DOI: 10.2478/romneu-2014-0021

\title{
Intracranial pressure monitoring in severe traumatic brain injury
}

\author{
Al. Cosmin Apetrei ${ }^{1,2}$, A.Şt. Iencean ${ }^{2}$, A Iordache I,2 $^{1,2}$ B Iliescu ${ }^{1,2}$, \\ Ion Poeata ${ }^{1,2}$
}

${ }^{1} \mathrm{PhD}$ Student "Grigore T. Popa" University of Medicine and Pharmacy Iasi ${ }^{2}$ Department of Neurosurgery, Emergency Hospital "Prof. Dr. N. Oblu Iasi”

\begin{abstract}
Intracranial pressure monitoring seems to be an indispensable stage in management of severe traumatic brain injured patient. Since 2009, this technique completes our trauma protocol. The study has been carried out from 2011 to 2013 in Prof. Dr. N. Oblu hospital in Iasi. There have been included in the study patients with severe craniocerebral trauma, who had traumatic brain lesions CT detected and Glasgow score between 3 and 8 . The age ranged from 16 to 60 , an average of 35.5 years old. $50 \%$ of the studied cases had a favorable outcome. Diagrams associated to this category of patients showed increases in intracranial pressure above normal values but without repeated values above $50 \mathrm{~mm} \mathrm{Hg}$. Most of those patients had a good evolution under medical treatment. Monitoring intracranial pressure is an extremely useful stage in treating intracranial high pressure in traumatology and it should be included in the equipment of any intensive therapy section caring traumatic patients.
\end{abstract}

Key words: brain edema, intracranial pressure, severe traumatic brain injury.

\section{Introduction}

Despite modern medicine advances in brain traumatology, a problem with important socio-economic and family consequences still remains. It is the main death cause with people under 45 in the United States and Europe. [1]

In the United States about 1,7 million cases of craniocerebral injuries are reported every year [2] among which almost $25 \%$ are patients needing hospitalization. The estimated costs required for caring them amounts to 60 billions dollars a year in the United States. [3]

Monitoring intracranial pressure with patients suffering from severe traumatic brain injury is recommended by Brain Trauma Foundation Guides. Since the 60s intracranial high pressure has been intensely studied as a predictive factor in the evolution of those with severe craniocerabral injury. At the same time knowing intracranial pressure helps precocious detection of intracranial high pressure as well as finding appropriate treatment for it. 
Monitoring intracranial pressure is recommended with all patients suffering from serious brain injury and having Glasgow score between 3 and 8 and CT pathological investigation (hematoma, edema, contusion, compression of basal cisterns).

Patients with severe brain injury and normal CT, if two or more than the following situations are recorded at admission: more than 40 years old, unilateral or bilateral motor deficiency, systolic blood pressure under $90 \mathrm{~mm}$, are also recommended monitoring.

Analysis of studies carried out since the 70s have revealed that patients having benefitted from monitoring their intracranial pressure recorded $12 \%$ lower mortality. [4]

Intracranial pressure can be measures through invasive or noninvasive methods. Measuring systems with intracranial pressing transducer are: intraventricular, intraparenchimatous, subdural, epidural.[5] All these devices have been used in our study.

\section{Material and methods}

A group of 16 patients with severe brain injury (CGS 3-8) have been studied. Their intracranial pressure has been permanently been monitored through invasive method. The study has been carried out from 2011 to 2013 in Prof. Dr. N. Oblu hospital in Iasi.

There have been included in the study patients with severe craniocerebral trauma, who had traumatic brain lesions CT detected and Glasgow score between 3 and 8 . The age ranged from 16 to 60 , an average of 35.5 years old. Intracranial pressure captor was set between 0 and 48 hours since presentation according to $\mathrm{CT}$ examination and clinic condition. Intraventricular, intraperenchimatos or subdural captors were used, as the case required. In some cases all models compatible with Camino MPM 1 SDC system produced by Integra had to be used (Figure 1). Patients suffering from other serious life threatening lesions, needing emergency treatment in other clinics, with severe coagulation troubles, dying people were not included in the program.

Data have been collected and worked out with the help of MPM 1 RS 232 Interface Software Program.

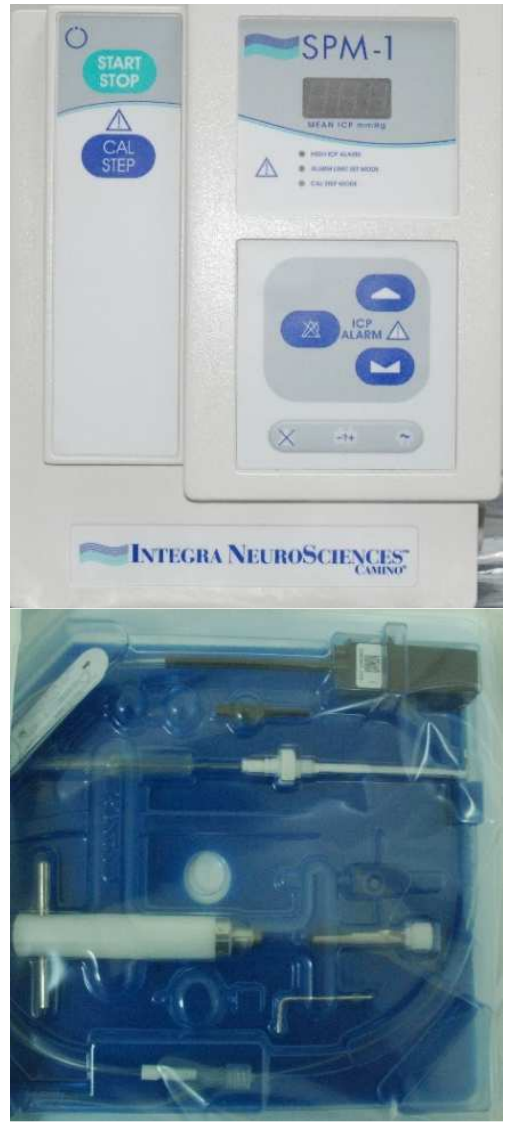

Figure 1 - Intracranial pressure monitor and intraventricular bolt 
DOI: 10.2478/romneu-2014-0021

\section{Results}

Between 2011 and 201316 patients with serous traumatic brain injury were invasively monitored. All were CT examined when hospitalized and after setting the implant none developed neither hemorrhagic complications nor malpositionnment.

The present study is not meant to present individual cases from the viewpoint of the treatment methods effectiveness but it establishes correlations between certain types of increasement of intracranial pressure and the patient's evolution. 8 out of 16 monitored patients died despite upmost therapy, four of them benefitted from decompressive craniectomy.

The cases were divided into 2 lots according to the evolution and namely those who deceased and those who were discharged. In the first lot it was recorded that the age average was higher (37.5) than in the second (33.5), the age limits being 16 to 60 . We could note that the only complication occurred was pneumonia in 3 cases, $18.75 \%$, and that with patients with long hospitalization.

The graphic representation given by the monitor soft produced by Integra allowed us to identify two pressure variation patterns: diagrams containing repeated spikes of above $50 \mathrm{~mm} \mathrm{Hg}$ (the utmost value the soft can represent) (Figure 2), were associated to the patient's unfavorable evolution in all monitored cases. The second pattern where even pathologic pressure were not accompanied by sudden high increases were associated to patients having favorable evolution. (Figure 3)

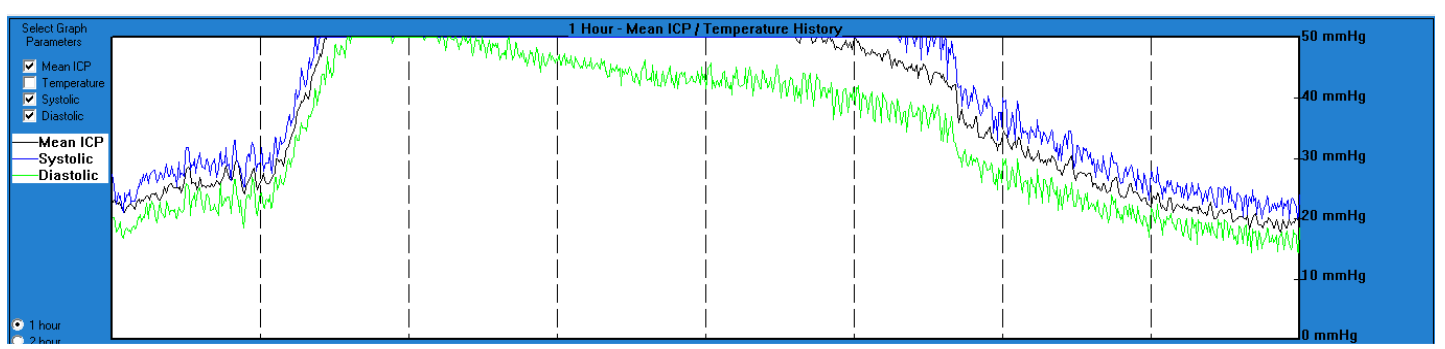

Figure 2 - Values more than $50 \mathrm{mmHg}$ associated with poor prognosis

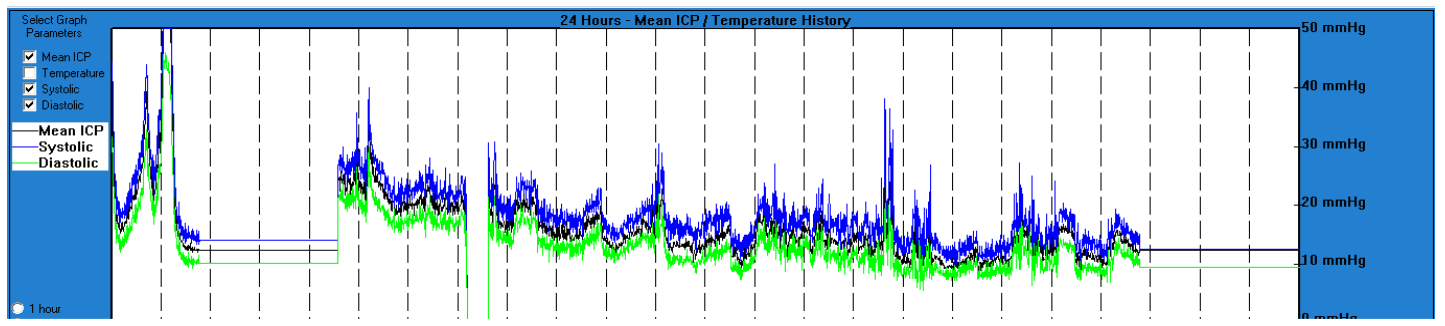

Figure 3 - Rare spikes, normal values, good prognosis 


\section{Discussions}

Monitoring intracranial pressure with patients having serious traumatic brain injury is not a new method of managing treatment and it is continually progressing due to technological advances.

Alexander Monro and his student George Kellie proposed the concept of intracranial pressure in the 18th century. The doctrine bearing their names (Monro-Kellie) states the cranial compartment is not an extendable container, so the volume is constant. The content made of brain tissue, blood, cefalorahidian liquid has a volume balance so that any increase in a component's volume must be compensated by another component's volume decrease.

1891 - Quinke published the first studies on lumbar puncture. He added a dropper at the end of the needle which measures pressure. [6].

1965 - Lundberg revolutionized monitoring intracranial pressure using manometers for continuous registering by ventriculostomy.

At present there are several guides for cranio-cerebral traumatology. In the case of severe traumatic brain injuries the most popular guide is that of Brain Trauma Poundation, "Guidelines for the Management of Severe Traumatic Brain Injury 3rd Edition", accepted by the hospital where this research has been done. As compared to the second edition, the recommendation levels were modified and namely, from standard, guideline and option to level I, level II and level II. These levels derive from evidence classes I,
II, III.

According to this guide, intracranial pressure should be monitored with all patients suffering from severe traumatic brain injury (CGS 3-8 after resuscitation), having abnormal CT examination. Abnormal craniocerebral CT means hematoma, contusions, swelling, herniations and basal tank compression. Intracranial pressure monitoring is indicate with patients suffering from severe brain trauma with normal CT examination if at presentation at hospital they have two or several of the following: more than 40 years old, uni or bilateral motor deficiency, systolic blood pressure lower than $90 \mathrm{~mm} \mathrm{Hg}$.

We had noticed that $50 \%$ of the studied cases had a favorable outcome. Diagrams associated to this category of patients showed increases in intracranial pressure above normal values but without repeated values above $50 \mathrm{~mm} \mathrm{Hg}$. Most of those patients had a good evolution under medical treatment. Decompressive cranietomy was required in only 2 out of 8 cases.

The diagram pattern of those evolving to multiple spikes above $50 \mathrm{~mm} \mathrm{Hg}$ values of intracranial pressure which persisted after aggressive antidedematous treatment. Four out of the eight deceased had undergone decompressive cranietomy. Two of them were subject to important postoperative intracranial pressure with multiple values above $50 \mathrm{~mm} \mathrm{Hg}$ refractory to any available therapeutic attitude.

This study demonstrates that a certain type of raised intracranial pressure represents a factor for the patient's prognosis. Repeated increases of values above $50 \mathrm{~mm} \mathrm{Hg}$ 
DOI: 10.2478/romneu-2014-0021

irresponsive to aggressive treatment for intracranial high pressure correlates to poor outcome $100 \%$ of the cases.

Taking into account this aspect a question arises: should aggressive treatment continue with patients whose diagram suggests an unfavorable prognosis or a supportive therapy is more adequate? The patients being comatose, therapeutic decision shall be taken in agreement with the family, according to the legislation in force, a first degree relative.

\section{Illustrative case}

A 25 year old patient comes to hospital in pre comatose condition, CGS 9, after having been aggressed and suffered a brain trauma subsequent to fall. 30 hours after admission his condition worsens to CGS 7, therefore the CT examination is repeated and an intracranial pressure captor is set.

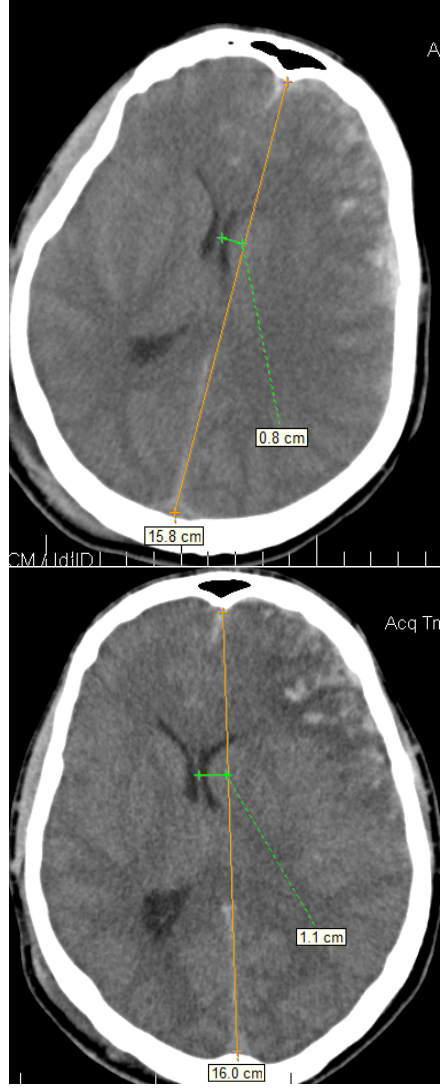

Figure 4 - CT examination of presented case: (left) at admission and (right) 30 hours later after neurological deterioration

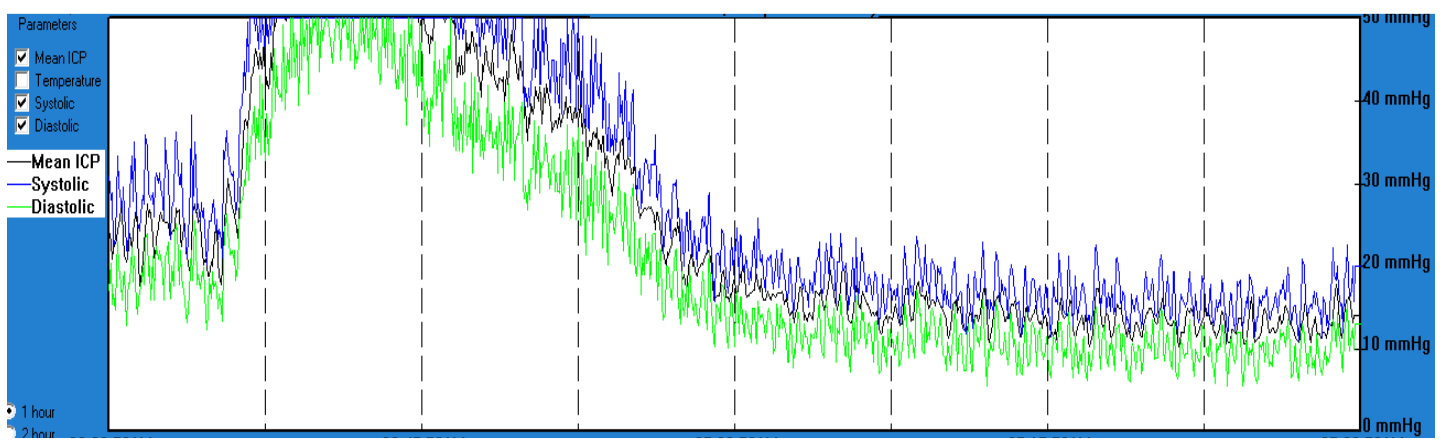

Figure 5 - Diagram of intracranian pressure showing significant pressure decrease o after manitol and continuous sedation 
Measures are taken in order to treat the diffuse brain edema with manitol and sedation with propofol - good evolution (Figure 5)

12 hours later he becomes reactive to drug treatment and an unilateral left decompressive cranietomy is carried out. (Figure 6)

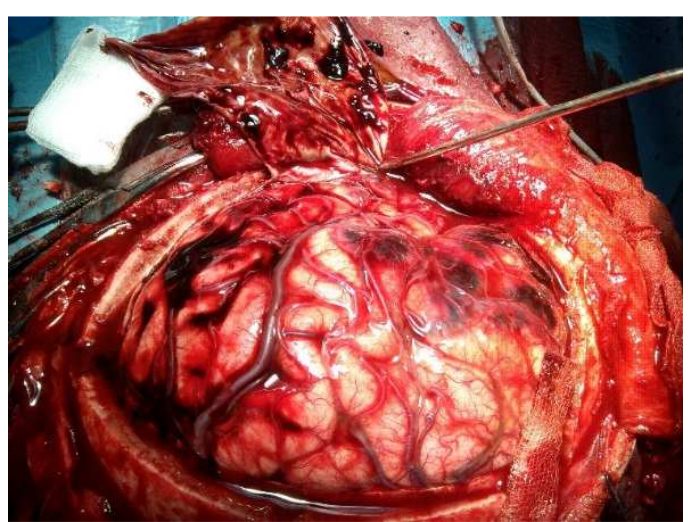

Figure 6 - Intraoperative aspect of left decompressive craniectomy at presented patient
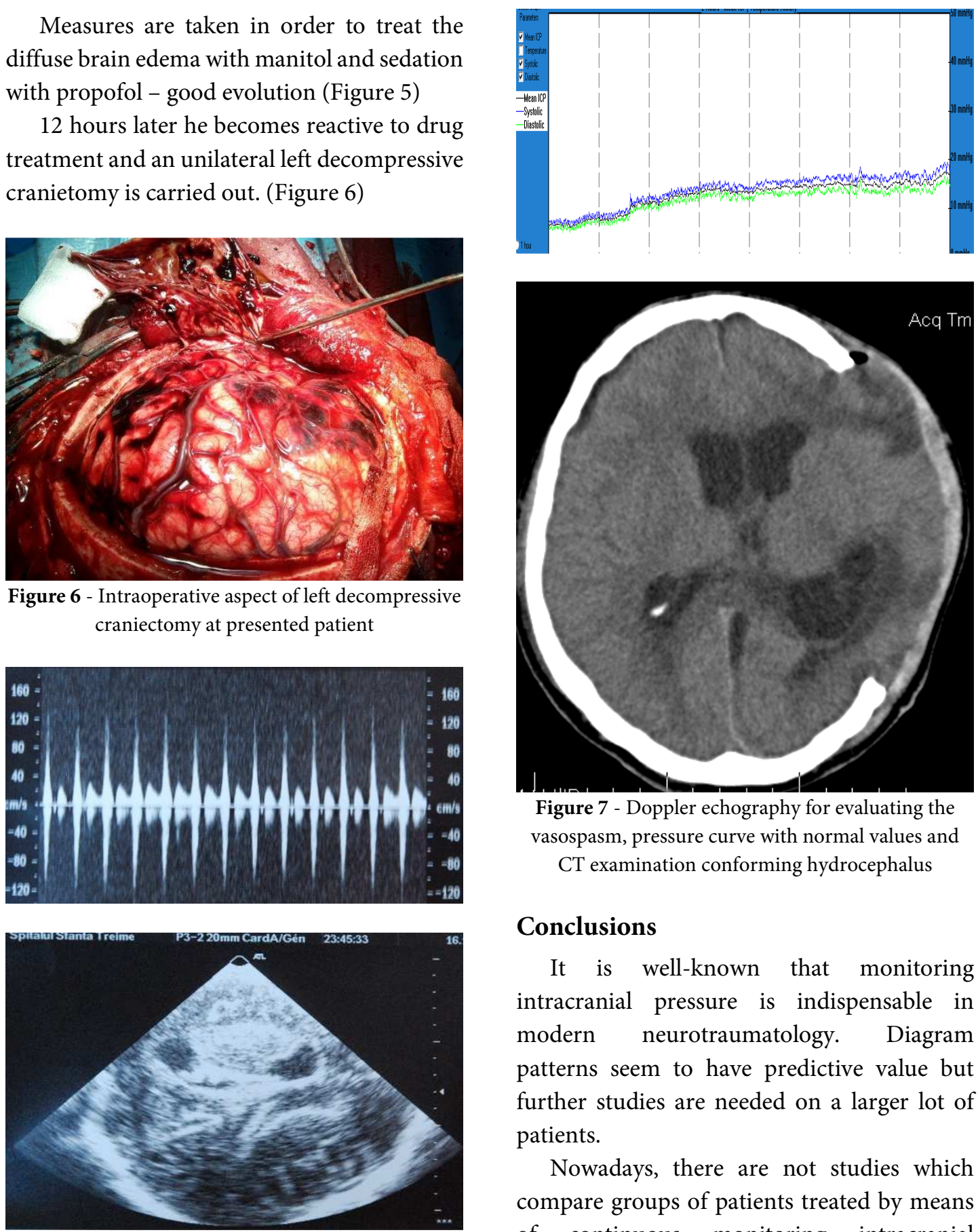

Figure 7 - Doppler echography for evaluating the vasospasm, pressure curve with normal values and CT examination conforming hydrocephalus

\section{Conclusions}

It is well-known that monitoring intracranial pressure is indispensable in modern neurotraumatology. Diagram patterns seem to have predictive value but further studies are needed on a larger lot of patients.

Nowadays, there are not studies which compare groups of patients treated by means of continuous monitoring intracranial 
DOI: 10.2478/romneu-2014-0021

pressure, to groups of patients who didn't benefit from it.

This study completed on a small group of patients confirm the present data. Decompressive craniectomy decreases intracranial pressure. Monitoring intracranial pressure is an extremely useful stage in treating intracranial high pressure in traumatology and it should be included in the equipment of any intensive therapy section caring traumatic patients.

\section{References}

1. Hyder AA, Wunderlich CA, Puvanachandra P, Gururaj G, Kobusingye OC: The impact of traumatic brain injuries: a global perspective. NeuroRehabilitation 22:341-353, 2007)

2. JNS iunie 2013 pag 1306

3. Finkelstein EA, Corso PS, Miller TR, The Incidence And Economic Burden of Injuries in the United States, New York: Oxford University Press, 2006)

4. Stein et all. Relationship of aggressive monitoring and treatment to improved outcomes in severe traumatic brain injury. Clinical article. J. Neurosurg 112: 1105-1112, 2010.

5. Iencean St. M. Hipertensiunea intracranaiana. Institutul European 2006

6. Quincke H. Ueber Hydrocephalus. Congresses für Innere Medizin, Zehnter Congress. 1891;10:321-331.

7. C. Werner ${ }^{\star}$ and K. Engelhard, Pathophysiology of traumatic brain injury British Journal of Anaesthesia 99
(1): 4-9 (2007) doi:10.1093/bja/aem131

8.Lobato RD, Cordobes F, Rivas JJ, et al. Outcome from severe head injury related to the type of intracranial lesion. A computerized tomography study. J Neurosurg. 1983;59:762.

9. Spencer WH. On the Changes Produced in the Circulation and Respiration by Increase of the Intracranial Pressure or Tension. Proceedings of the Royal Society of London. Proceedings of the Royal Society of London. 1890;48:273-275.

10. (Winn, H. Richard (2011-06-10). Youmans Neurological Surgery E-Book (Kindle Location 284640). Elsevier Health. Kindle Edition.)

11. Cerebral Vasospasm in Traumatic Brain Injury Daniel R. Kramer, Jesse L. Winer, B. A. Matthew Pease, Arun P. Amar, and William J. Mack Department of Neurosurgery, University of Southern California, 1200 North State Street, Suite 3300, Los Angeles, CA 90033, USA Received 29 October 2012; Accepted 4 June 2013

12. Czosnyka M, Hutchinson PJ, Balestreri M, Hiler M, Smielewski P, Pickard JD. Monitoring and interpretation of intracranial pressure after head injury. Acta Neurochir Suppl. 2006;96:114-8.

13. Crippen D. Head Trauma. Medscape Reference. Available

http://emedicine.medscape.com/article/433855overview.

14. Friedman DI, Jacobson DM. Diagnostic criteria for idiopathic intracranial hypertension. Neurology. Nov 26 2002;59(10):1492-5

15. Spencer WH. On the Changes Produced in the Circulation and Respiration by Increase of the Intracranial Pressure or Tension. Proceedings of the Royal Society of London. Proceedings of the Royal Society of London. 1890;48:273-275 